which test for STIs provide key epidemiologic data, identify gaps, and should guide policies.

Methods PrEP projects are reaching populations at risk of HIV in LMIC, including adolescent girls and young women (AGYW), heterosexual HIV serodiscordant couples, and men who have sex with men. STI data from different populations and geographies will be reviewed, and research and intervention gaps will be discussed.

Results Among African AGYW in PrEP projects, the prevalence of Chlamydia trachomatis (CT) and/or Neisseria gonorrhoea (GC) is $30 \%$, most of which is asymptomatic, syphilis is $<1 \%$, and annual incidence of CT is $30 \%$ and GC is $10 \%$, most of which are new infections. Among African heterosexual HIV serodiscordant couples in PrEP studies, the prevalence of CT is $10 \%$, trichomonas is $7 \%$, GC is $6 \%$, and syphilis is $<1 \%$. STI prevalence among African men who have sex with men (MSM) are more sparse, but indicate high prevalence of GC, CT, and syphilis.

Conclusion The prevalence of curable bacterial STIs is high in PrEP projects among diverse populations in LMIC, most of which are missed by syndromic STI management. STI services are an important reproductive sexual health intervention, are valued by clients, and increase the public health impact of PrEP. Interventions to lower the costs of STI diagnostic assays, point of care tests, and treatment of partners are needed. Innovative STI prevention strategies should be evaluated, including doxycycline post-exposure prophylaxis and vaccines. STI testing, treatment, and partner services should be integrated into PrEP, medical male circumcision, antenatal care, and HIV care and prevention programs which reach sexuallyactive persons in LMIC.

Disclosure No significant relationships.

\section{S10.2 BUILDING STD CLINICAL INFRASTRUCTURE IN LMIC - ROLE OF GENERAL VS. SPECIALIZED INFRASTRUCTURE}

Ligang Yang*. Dermatology Hospital, Southern Medical University, STD Dept, Guangzhou, China

\subsection{6/sextrans-2019-sti.50}

Making clinical service for STD patients accessible in LMICs will require to recognize common medical conditions. In China, general hospitals are the most visited by patients, including those with STD. Data from 105 Chinese STD sentinel sites in 2018 showed that $77.2 \%$ of syphilis cases were reported from general hospitals, followed by $5.6 \%$ from women and children hospitals, only $4.7 \%$ of syphilis cases from STD specialized institutions. Community health centers, although providing comprehensive medical services, are generally considered to be of lower level and only 3.0\% syphilis cases were rereported from the community health centers and other primary care centers. In LMICs with limited human resources, general hospitals play very important role in providing clinical services for STD patients but are often unsatisfactory in preventive services. A survey from China found, the rates of contact tracing and partner notification were very low in general hospital as the physicians lack time and willingness for preventive services. The main function for general hospitals, women and child health care hospitals, are to provide STD screening and corresponding treatment for out-patients, inpatients and pregnant women. As for the specialized STD institutions, it usually includes the following functions:1.
Develop appropriate local STD case management procedures (flow chart); 2. STD surveillance, including case auditing and active surveillance; 3 . STD testing center including external STD lab quality assessment; 4. Young people clinics, MSM clinics, et al; 5. Training. To make these services effective, integration of general and specialized institutions at the managerial and administrative levels will be crucial.

Disclosure No significant relationships.

\section{S10.3 CERVICAL CANCER PREVENTION IN LMICS: ARE WE ON THE PATH TO ELIMINATION?}

Megan Huchko*. Duke University, Durham, USA

10.1136/sextrans-2019-sti.51

In May 2018, the World Health Organization announced that a cosmmitment to cervical cancer elimination. In October 2018, modelers from Australia showed that with current rates of vaccine implementation and screening, cervical cancer could be eliminated in that country within the next ten years. Researchers are working on clinical, implementation and modeling studies to determine the most effective strategies to achieve cervical cancer elimination. We examined prior commitments to disease elimination, modeling data on public health strategies for cervical cancer elimination in high and low-income countries, and possible challenges to successfully achieving elimination in the world's most vulnerable populations. Cervical cancer elimination, defined as fewer than four cases per 100,000 women, could be achievable in high-income countries (HICs) within the next 25 to 50 years. The speed and ultimate success of elimination is driven by high rates of coverage with the 9-valent vaccine, with screening providing only a small contribution. The most effective models in lowand middle-income countries (LMICs) include targeting female adolescents for two-dose vaccination, followed by either one or two lifetime screens. Challenges include a global shortage of the 9-valent vaccine, slower than anticipated roll-out to GAVI countries and low uptake rates in countries with vaccination programs. Remarkable advances in biomedical technologies have made cervical cancer elimination a potentially attainable goal within the next generation. Focusing efforts on widespread and effective implementation of evidence-based strategies to prevent the disease will be crucial. We must work to ensure that progress toward elimination is shared equally between HICs and LMICs to avoid a perpetuation of the global health disparity seen in cervical cancer.

Disclosure No significant relationships.

\section{S10.4 SELF-CARE INTERVENTIONS FOR SEXUAL AND REPRODUCTIVE HEALTH AND RIGHTS}

Manjulaa Narasimhan*. World Health Organization, Geneva, Switzerland

\subsection{6/sextrans-2019-sti.52}

Among the most promising and exciting new approaches to reach Universal Health Coverage (UHC) goals are self-care interventions for health. These interventions have the potential to increase choice, when accessible and affordable, as well as opportunities for individuals to make informed decisions regarding their health and health care. Self-care interventions 
recognize the strengths of individuals as active agents in their health, and not merely passive recipients of health services.

To support WHO normative guideline on innovative selfcare strategies for sexual and reproductive health and rights (SRHR), a conceptual framework was developed and the evidence base was evaluated on interventions in transition from provision by facility-based, healthcare providers to delivery in the self-care environment, including STI and HPV self-sampling. While further research is needed on user understanding, uptake and access, our reviews suggest that STI and HPV selfsampling are effective strategies to increase STI and HPV testing uptake respectively.

Challenge remain, however, for vulnerable, marginalized and socioeconomically underprivileged populations, who have the poorest health outcomes globally, to access quality health innovations, including self-care interventions. The WHO guideline on self-care interventions for SRHR is framed around key principles of human rights, gender equality and a holistic, peoplecentred approach to health and well-being. The potential benefits of such an approach includes the creation of a safe and supportive enabling environment which can potentially increase health coverage, access and quality of services; and thereby reduce health inequities. There is already widespread and rapidly growing use of self-care interventions. When these interventions are people-centred, and evidence-driven, even vulnerable populations will be able to exercise their rights to health, to information, to autonomous decision-making. Disclosure No significant relationships.

\section{S11 - RESEARCH ON THE VAGINAL MICROBIOME: ADVANCES \& CONTROVERSIES}

\section{Tuesday, July 16, 2019 10:45 AM - 12:15 PM}

\section{S11.1 INTRODUCTION TO THE VAGINAL MICROBIOME PRE- CONFERENCE SYMPOSIUM}

Jeanne Marrazzo*. University of Alabama, Medicine, Birmingham, USA

10.1136/sextrans-2019-sti.53

This will comprise an introduction to this session. Disclosure No significant relationships.

\section{S11.2 LESSONS LEARNED FROM THE PRE-MEETING SYMPOSIUM ON CHARACTERIZING THE VAGINAL MICROBIOTA THROUGH A BLINDED MULTI- LABORATORY COLLABORATION}

Jennifer Balkus*. University of Washington, Department of Epidemiology, Seattle, USA

10.1136/sextrans-2019-sti.54

In studies of the microbiome, variability can arise from numerous steps in the microbial profiling process, including DNA extraction, PCR amplification, and bioinformatics approaches for taxonomic assignment. This variability may contribute to issues with reproducibility in clinical and epidemiologic studies. Others have described the contribution of different steps in the microbial profiling process to measurement variability in samples comprised of bacteria from the human gut. We conducted a comparative study across four laboratories to better understand sources of variation in describing the human vaginal microbiota. In this session, we will share highlights from the pre-conference symposium that presented lessons learned from this comparative project and discuss implications for future vaginal microbiota research.

Disclosure No significant relationships.

\section{S11.3 THE VAGINAL MICROENVIRONMENT PRIOR TO INCIDENT STI}

Rebecca Brotman*. University of Maryland School of Medicine, Institute of Genome Sciences, Baltimore, USA

\subsection{6/sextrans-2019-sti.55}

We sought to evaluate microenvironmental factors in the vagina that drive protection against STIs. We conducted a nested case control study in the Longitudinal Study of Vaginal Flora to assess the vaginal microenvironment of 397 cases at the visit before an incident genital STI (Chlamydia trachomatis, Neisseria gonorrhea, or Trichomonas vaginalis) and 1,794 STI-negative controls. Controls were matched to cases on age, race and follow-up time. Vaginal lavages and surveys were collected every three months for one year. Vaginal microbiota, metagenomes, metabolites, and lactic acid isomers were assessed as factors associated with incident STI. Bacterial community state types (CSTs) were assigned by hierarchical clustering of vaginal microbiota. Metagenomes of 708 participants were characterized using VIRGO. We used conditional logistic regression with covariate adjustment (partner concurrency, number of sex partners, condom use). Women with a CST-IV-A profile, low-Lactobacillus with high relative abundance of BVAB-1, had the highest odds of incident STI. CST-I (L. crispatus-dominated), CST-II (L. gasseri-dominated), CST-III-A, and CST-III-C (both $L$ iners-dominated; the latter has other Lactobacillus spp.) had $>50 \%$ lower odds of STI than women in CST IV-A (all $\mathrm{p}<0.01$ ). CST-II had the lowest point estimate $(72 \%$ lower odds, $\mathrm{p}=0.02)$. Metagenomic analyses confirmed these findings and revealed a cluster of G. vaginalis sub-species with $40 \%$ lower odds of STI than the BVAB-1 dominated cluster $(\mathrm{p}=0.02)$. Higher bacterial absolute abundance had lower odds of STI in each CST $(\mathrm{p}<0.001)$. High D-lactic acid concentration was associated with lower STI, irrespective of L-concentration $(\mathrm{p}<0.05)$. Of the 185 metabolites that were significantly associated with incident STI (q-value $<0.05$ ), 13 metabolites, including taurine and kynurenine (microbial metabolites with immunomodulating properties), were associated with $\geq 20 \%$ lower odds of STI per fold change. Multi-omic interrogation revealed protection against STI acquisition was associated with vaginal microenvironments containing immunomodulatory metabolites, as well as Lactobacillus spp. that produce D-lactic acid. Disclosure No significant relationships. 